\title{
Estimación de los esfuerzos generados en el hombro por parte de trabajadores de la industria del automóvil mediante modelos biomecánicos de medidas in situ
}

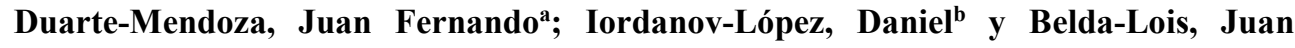 \\ Manuel $^{b}$ \\ ${ }^{a}$ Universitat Politècnica de València (UPV), Valencia, España. juaduame@etsii.upv.es, \\ ${ }^{\mathrm{b}}$ Instituto de Biomecánica (IBV), Universitat Politècnica de València (UPV), Valencia, España. \\ daniel.iordanov@,ibv.org,jmbelda@ibv.org
}

\begin{abstract}
Linear work in the automotive industry involves risks derived from repeated tasks and forced postures maintained over time. Although there are several platforms and tools that try to ergonomically evaluate jobs, such as the risk assessment software REBA and ERGO / IBV, these tools lack sensitivity when determining aspects related to the risk of injury. This happens especially when new aids are introduced in the workplace, different from the usual methods, which are not contemplated, such as the use of exoskeletons. That is why we present a methodology to estimate the muscular and joint efforts associated with linear work. We evaluate, ergo, 10 workers doing their usual work in real conditions. A superior limb model implemented in OpenSim is used, to which the dynamics of the movements measured with inertial sensors and the registration of the muscular signal of four muscles are introduced: anterior aspect of the deltoid, portion upper trapezius, the spinal erector and the large dorsal. The results obtained show a good consistency between the recorded muscle estimates and those estimated by the biomechanical model. These results can lay the practical basis for an objective assessment of risk in the workplace with novel conditions such as the use of exoskeletons.
\end{abstract}

Keywords: biomechanics models, effort estimation, exoskeletons.

\begin{abstract}
Resumen
Los trabajos lineales en la industria del automóvil involucran riesgos derivados de tareas repetidas y posturas forzadas mantenidas en el tiempo. Si bien, varias son las plataformas y herramientas que intentan evaluar ergonómicamente los puestos de trabajo, como los software de valoración de riesgos REBA y ERGO/IBV, estas herramientas carecen de sensibilidad a la hora de determinar aspectos relacionados con el riesgo de la lesión. Esto ocurre especialmente cuando se introducen nuevas ayudas en los puestos de
\end{abstract}


Estimación de esfuerzos generados en el hombro por parte de trabajadores de la industria del automóvil mediante modelos biomecánicos de medidas in situ

trabajo, distintas a los métodos habituales, que no están contempladas, como el uso de exoesqueletos. Es por ello que se presenta una metodología para estimar los esfuerzos musculares y articulares asociados al trabajo lineal. Evaluamos, ergo, a 10 trabajadores realizando su trabajo habitual en condiciones reales. Se utiliza un modelo de miembro superior implementado en OpenSim, al que se le introduce la dinámica de los movimientos medidos con sensores inerciales y con el registro de la señal muscular de cuatro músculos: cara anterior del deltoides, porción superior del trapecio, el erector espinal y el gran dorsal. Los resultados obtenidos muestran una buena consistencia entre las estimaciones musculares registradas y las estimadas por el modelo biomecánico. Estos resultados pueden sentar las bases prácticas para una valoración objetiva del riesgo en el puesto de trabajo con condiciones novedosas tal como el uso de exoesqueletos.

Palabras clave: modelos biomecánicos, estimación de esfuerzos, exoesqueletos.

\section{Introducción}

La industria del automóvil siempre ha sido uno de los sectores más importantes en el desarrollo y progreso de la economía de un país, no solo por su relevancia y aportación a la economía y al empleo, sino también por su capacidad de impulsar otros sectores. En España, supone ser un pilar estratégico, pues es uno de los principales propulsores de inversión y desarrollo en términos de innovación, seguridad y protección del medio ambiente, contribuyendo de manera significativa al desarrollo del país de forma directa, indirecta e incluso inducida. (Ramírez, 1993).

Su verdadera importancia radica, especialmente, en el hecho de que agrupa una serie de actividades de distinta naturaleza destacando las áreas encargadas de montaje de los productos fabricados. Es, justamente en estas áreas, donde se requieren esfuerzos mecánicos y movimientos forzosos por parte de los trabajadores para llevar a cabo las tareas requeridas. En muchas ocasiones dichas tareas con movimientos o posturas mantenidas en el tiempo superan los esfuerzos umbrales considerados normales, lo que puede conllevar la generación de trastornos musculo-esqueléticos en determinadas articulaciones. (Jonsson, 1982) La caracterización y comprensión de este tipo de tareas resulta esencial para mejorar las estrategias de ayuda enfocadas a la ergonomía y la reducción de riesgos en el trabajo. En España, este tipo de actividades que conllevan sobreesfuerzos suponen la afectación de más de 2500 personas de cada 100.000 trabajadores. (De Vicente, 2012). 
Para ello, hoy en día, se dispone de distintas herramientas que permiten obtener de alguna manera una primera valoración de los posibles esfuerzos que requiere un trabajo en concreto. Sin embargo, en la mayoría de ellas, lo que se evalúa simplemente es el análisis postural, en cuanto a aspectos ergonómicos se refiere, relacionado con el trabajo a realizar, dejando a un lado la valoración de un posible riesgo de lesión que éste pudiese generar. Estas herramientas suelen presentar una alta generalidad aplicable en muchas ocasiones, pero que implica que tengan una baja sensibilidad, es decir, los resultados obtenidos pueden ser pobres en detalles. Algunas de ellas, como el caso de los métodos REBA o RULA, han sido desarrolladas para la evaluación de las condiciones de trabajo (carga postural) estimando el riesgo de padecer desórdenes corporales en el trabajo (Hignett, 2000) o para investigar la exposición individual de los trabajadores a factores de riesgo de padecer transtornos musculoesqueléticos del miembro superior (Félix, 2011), respectivamente; o la aplicación ERGO/IBV - SOFTWARE EVALUACIÓN DE RIESGOS ERGONÓMICOS, diseñada para la evaluación y recomendación de diseño asociado a los riesgos ergonómicos y psicosociales de un puesto de trabajo. (ERGO/IBV - SOFTWARE EVALUACIÓN DE RIESGOS ERGONÓMICOS).

De igual manera sucede con la introducción de nuevos tipos de ayudas técnicas, novedosas con respecto a las técnicas clásicas, en gran parte debido al actual avance tecnológico, pero que no se encuentran contempladas en dichas herramientas de valoración funcional, como es el caso de los exoesqueletos.

Por otro lado, las técnicas de valoración que permiten obtener otras características de los movimientos realizados en las tareas del trabajo, como los ángulos relativos de los segmentos corporales, la cinemática de los mismos o incluso las fuerzas estimadas que llevan a cabo los músculos que forman parte de ellos, suponen ser herramientas que complementan a las de valoración funcional existentes, ayudando a potenciar la evaluación y determinación de los riesgos en el trabajo.

En este trabajo se describe una metología para la obtención de estimaciones de esfuerzos realizados en este tipo de trabajo por los grupos musculares implicados en la articulación del hombro o articulación glenohumeral: manguito de los rotadores (subescapular, infraespinoso, supraespinoso y redondo menor), trapecio y deltoides. Supone ser de las articulaciones más afectadas por el tipo de movimientos realizados, concretamente en aquellos en los que el brazo se encuentra por encima de la altura del hombro. Esto se debe a que se trata de una articulación holgada y poco profunda siendo la que mayor amplitud de movimientos permite, pero que a su vez es la que más luxaciones presenta. Anatómicamente, es una estructura articulada que sacrifica resistencia y estabilidad para obtener la gran amplitud de movimientos, necesarios para llevar a cabo diversas funciones. 
Estimación de esfuerzos generados en el hombro por parte de trabajadores de la industria del automóvil mediante modelos biomecánicos de medidas in situ

Se utilizan modelos biomecánicos que simulan la situación medida de la actuación del trabajador y se proporcionan las estimaciones de los esfuerzos que lleva consigo.

\section{Metodología}

Este trabajo propone un método para la estimación de esfuerzos realizados por los músculos implicados en la articulación del hombro. El primer paso consiste en la obtención de un modelo biomecánico adecuado del sujeto al que se desea evaluar en OPENSIM, con el que se puedan simular las medidas obtenidas para el mismo. Luego, se realiza un análisis cinemático inverso y un análisis dinámico inverso del modelo para un tiempo considerado en la toma de medidas. Por otra parte, se lleva a cabo un proceso de optimización estática con el que se obtendrán las estimaciones de los esfuerzos realizados por los distintos músculos.

\subsection{OpenSim: estimación de esfuerzos}

OPENSIM consiste en un software de código abierto que permite a los usuarios desarrollar modelos de estructuras musculoesqueléticas y crear simulaciones dinámicas de movimiento. (OPENSIM DOCUMENTATION) Con esta herramienta se consigue llevar a cabo la simulación y estimación deseada. De esta manera, se parte de un modelo de interés, concretamente aquel que simule de la forma más fidedigna posible y simplificada los movimientos de los miembros superiores, para así poder llevar a cabo un análisis de lo que sucede en la articulación del hombro. Se dispone de un conjunto de medidas de operarios en línea en condiciones reales que suponen ser la entrada del modelo.

\subsubsection{Escalado del modelo}

Se realiza el escalado del modelo para que presente propiedades semejantes y coincidentes con el sujeto de prueba, llevando a cabo una modificación de su antropometría. Para ello, OPENSIM ajusta las características de la masa en $\mathrm{kg}$ del sujeto y tiene en cuenta una serie de marcadores y segmentos de cuerpos rígidos definidos, 14 y 6 respectivamente, para el dimensionamiento del cuerpo. Se trata de un escalado basado en la medición, en la que se determinan los factores de escala para cada segmento mediante la comparación de puntos de referencia (marcadores virtuales y experimentales).

Se utiliza un conjunto marcadores y segmentos. Estos segmentos se corresponden con un cuerpo rígido del modelo y cuyos extremos están delimitados por los marcadores. Esto se puede apreciar en la Tabla 1. 


\subsubsection{Análisis cinemático inverso}

Con el modelo escalado se lleva a cabo un análisis cinemático empleando el toolbox de OPENSIM. Con este análisis, se consiguen los ángulos articulares del modelo musculoesquelético que mejor se ajustan a la cinemática experimental del sujeto. El problema de cinemática se resuelve para cada instante de tiempo (fotograma).

Tabla 1. Segmentos

\begin{tabular}{cccc}
\hline Nombre del segmento & Extremo distal & Extremo proximal & $\begin{array}{c}\text { Parte del modelo a la que se } \\
\text { asociada }\end{array}$ \\
\hline Brazo_d & pRightArmLatEpicondile & pRightAcromion & humerus \\
Brazo_i & pLeftArmLatEpicondile & pLeftAcromion & humerus_1 \\
Antebrazo_d & pRightUlnarStyloid & pRightArmMedEpicondile & ulna \\
Antebrazo_i & pLeftUlnarStyloid & pLeftArmMedEpicondile & ulna_1 \\
Hombro_d & pRightAcromion & pIJ & clavicle \\
Hombro_i & pLeftAcromion & pIJ & clavicle_1 \\
\hline
\end{tabular}

Con los resultados de la cinemática inversa resulta conveniente llevar a cabo una reducción de residuos, utilizando el algoritmo propio de OPENSIM para tal fin. Este algoritmo utiliza el modelo escalado y los resultados de la cinemática para realizar ajustes en las propiedades de inercia de los segmentos y la cinemática conjunta. Se obtiene, finalmente, un modelo y cinemática que son dinámicamente consistentes con las fuerzas que posteriormente se aplican.

\subsubsection{Análisis dinámico inverso}

Empleando el modelo escalado y los resultados de la cinemática se realiza un análisis dinámico del modelo empleando la correspondiente librería de OPENSIM, obteniendo la resolución de la estimación de fuerzas que afectan al modelo. Además, para este análisis resulta necesario añadir el valor de la fuerza que simula el peso de la herramienta que presenta el trabajador. Se crea de esta manera un fichero que presente dicho peso, una fuerza negativa en el eje y (según como se ha definido el modelo siguiendo los criterios de la ISB) con un valor de 2,5 $\mathrm{kg}$ que se corresponden con $24,5 \mathrm{~N}$, en una posición próxima a la mano con la que sujeta la herramienta. En este caso, se toma la situación de los marcadores de la mano derecha, pRightUlnarStyloid. 
Estimación de esfuerzos generados en el hombro por parte de trabajadores de la industria del automóvil mediante modelos biomecánicos de medidas in situ

Es necesario contar con los actuadores que participan en determinados movimientos (grados de libertad) del modelo y actúan como actuadores de reserva que conducen al modelo cuando no presenta determinados músculos o cuando la presencia de un conjunto de músculos es insuficiente para los requerimientos cinemáticos.

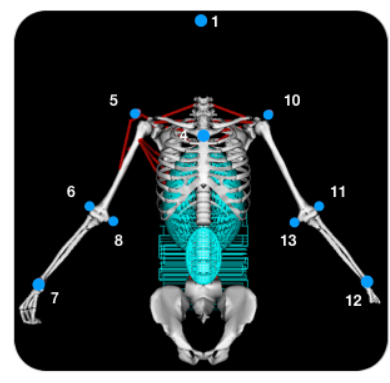

Vista anterior

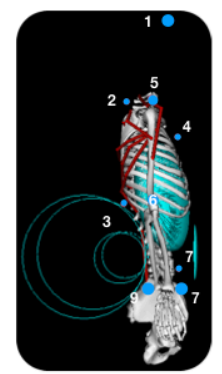

Vista lateral

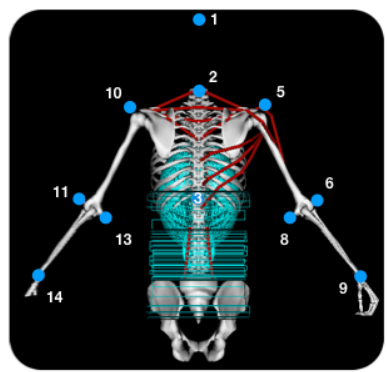

Vista posterior

\begin{tabular}{|c|c|c|}
\hline Marcadores derecha & Marcadores comunes & Marcadores izquierda \\
\hline 5. pRightAcromion & 1. pTopHead & 10. pLeftAcromion \\
\hline 6. pRightArmLatEpicondile & 2. pC7SpinalProcess & 11. pLeftArmLatEpicondile \\
\hline 7. pRightRadialStyloid & 3. pT12SpinalProcess & 12. pLeftRadialStyloid \\
\hline 8. pRightArmMedEpicondile & 4. pIJ & 13. pLeftArmMedEpicondile \\
\hline 9. pRightUlnarStyloid & - & 14. pLeftUlnarStyloid \\
\hline
\end{tabular}

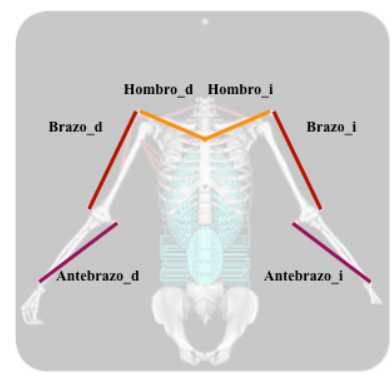

Fig. 1. Modelo "MoBL_ARMS_Upper_Limb": vista anterior, lateral y posterior, respectivamente. Marcadores. Segmentos definidos. Fuente: Creación propia (2019).

\subsubsection{Optimización estática}

Finalmente, la optimización estática se utiliza para abordar el problema de dinámica inversa resolviendo las ecuaciones de movimiento de las fuerzas generalizadas desconocidas que están sujetas a condiciones de activación de fuerza muscular y, así, poder estimar las activaciones y fuerzas musculares que las satisfacen.

Para una mayor simplificación del modelo, únicamente se consideran algunos músculos para el miembro superior derecho, entre los que se incluyen los involucrados en su articulación, y permitiendo los tres desplazamientos de la biomecánica del hombro: "elv_angle", "shoulder_elv" y "shoulder_rot", que se corresponden con los movimientos de aducción, flexo-extensión y rotación, respectivamente. 


\subsection{Modelo con muelle}

Toda la metodología anterior se aplica nuevamente sobre el mismo modelo, pero con una modificación de éste. En este caso, se introduce un resorte torsional en la articulación del hombro. Esta tipo de elemento de fuerza permite simular un resorte y un amortiguador sobre el rango de movimiento de una coordenada concreta, 'shoulder_elv', que se corresponde con el movimiento de flexión-extensión. Se simula un tope cuando la coordenada alcanza y supera su límite predefinido, es decir, su ángulo particular. El resorte añadido al modelo cuenta con las siguientes propiedades:

- Upper_stiffness: $-0.3 \mathrm{Nm} /$ grados

- Upper_limit: 5 grados

- Lower_stiffness: $1 \mathrm{Nm} /$ grados

- Lower_limit: -90 grados

- Damping: 0.00

- Transition: 2 grados

Donde estas características del elemento permiten la generación de fuerzas cuando el ángulo es mayor de $5^{\circ}$, con una activación de rigidez de $30 \mathrm{Nm} /$ grado, y una transición de 2 grados, cuando pasa de cero a $\mathrm{K}$ en el momento que la coordenada excede su límite. No se considera ningún tipo de amortiguación por lo que el damping debe estar a cero.

Con la adición del resorte se consigue simular el efecto de ayuda al movimiento que realizaría un exoesqueleto, en caso de que el trabajor lo llevase puesto.

\subsection{Comparación de los esfuerzos musculares}

De cada sujeto analizado, se obtiene la activación muscular y la estimación de fuerza muscular realizada por los distintos conjuntos de músculos estudiados, tanto para el modelo genérico como para el modelo que simula la existencia del exoesqueleto. En este paso, se pretende determinar si existen diferencias entre la presencia o ausencia del exoesqueleto, para un sujeto concreto en el mismo intervalo de tiempo, en algunas líneas de acción musculares consideradas relevantes por la posición del brazo en el movimiento analizado: TRAP1 (Trapecio), DELT2 y DELT3 (deltodides). Para ello, se distingue visualmente con las gráficas obtenidas para las fuerzas y se calculan los percentiles p25, p50 y p75, comparándolos entre ellos. 


\section{Materiales}

\subsection{Sujetos}

El planteamiento propuesto se aplicó sobre un total de 5 sujetos con exoesqueleto de un conjunto medidas in situ realizadas a un grupo de 12 trabajadores de la empresa de automóviles Ford Almussafes (Valencia), llevadas a cabo por el Instuto de Biomécanica de Valencia (IBV), de los cuales 6 trabajadores fueron considerados como casos y los otros 6 como controles, en función de si llevaban o no el exoesqueleto utilizado en las medidas, respectivamente. Todos los sujetos son varones sanos diestros, excepto uno de ellos que es una mujer, con alturas comprendidas entre 1,67 y 1,85 metros y pesos entre 64,4 y 83,3 kilogramos.

Para la obtención de las medidas se instrumentó a los sujetos con un sistema de EMG (electromiograma Ultium ${ }^{\mathrm{TM}}$ EMG) y un sistema de captura de movimiento (Xsens con el software MVN Analyze) basado en sensores inerciales, modelos biomecánicos y algoritmos de fusión de sensores. (XSENS MVN ANALYZE) Además, se les pidió a los trabajadores que llevaran a cabo dos test: uno de 3 ciclos del trabajo lineal realizado, que serviría como línea base (*para los casos considerados el test 1 es realizado sin exoesqueleto), y otro de 5 ciclos del trabajo lineal.

Tabla 2. Conjunto de medidas realizado

\begin{tabular}{c|cc}
\hline $\begin{array}{c}\text { Características de } \\
\text { la medida }\end{array}$ & $\begin{array}{c}\text { Controles (sin } \\
\text { exoesquelo) }\end{array}$ & $\begin{array}{c}\text { Casos (con } \\
\text { exoesqueleto)* }\end{array}$ \\
\hline $\mathrm{N}^{\mathrm{o}}$ sujetos & 6 & 6 \\
Test 1 & $\begin{array}{c}3 \text { ciclos de trabajo } \\
\text { (línea base) }\end{array}$ & $\begin{array}{c}3 \text { ciclos de trabajo (línea } \\
\text { base, sin exoesqueleto) }\end{array}$ \\
Test 2 & 5 ciclos de trabajo & $\begin{array}{c}5 \text { ciclos de trabajo (con } \\
\text { exoesqueleto) }\end{array}$ \\
\hline $\mathrm{N}^{\mathrm{o}}$ Medidas & 12 & 24 (2 días) \\
\hline
\end{tabular}

De las medidas realizadas se dispone directamente de los archivos ".tre" de las mismas en los que se encuentran las posiciones de todos los sensores a lo largo del tiempo. Se lleva a cabo, de esta manera, todo el proceso en 5 de los trabajadores casos del Test 2, que han realizado los 5 ciclos de trabajo con exoesqueleto. 


\subsection{Modelo Biomecánico}

El modelo biomecánico utilizado en OPENSIM, es un modelo simplificado del modelo original de "MoBL_ARMS_Upper_Limb_Model_OpenSim.osim", en el que se mantiene y destacan las componentes del tronco y miembros superiores, de acuerdo al objetivo del trabajo. Asimismo, el modelo modificado presenta los marcadores necesarios y rotulados de manera correcta, así como los músculos involucrados en el movimiento: dorsal latera, trapecio y deltoides. Cada músculo está representado por un múltiples líneas de acción dado que se trata de músculos extensos que tienen insersiones en extensiones grandes. Así:

- Dorsal lateral: LAT1, LAT2 y LAT3.

- Trapecio: TRAP1, TRAP2, TRAP3 y TRAP4.

- Deltoides: DELT2 y DELT3.

De la misma manera, se dispone del modelo “MoBL_ARMS_Upper_Limb_Model_OpenSim.osim" modificado, con la adición del muelle requerido para la simulación de portación del exoesqueleto.

\section{Resultados}

El tiempo de duración para cada una de las medidas de las que se dispone es alrededor de 5 minutos, donde se recoge todo el ciclo de trabajo: momentos en los que el brazo se sitúa por encima del hombro o alcanza su altura y el sujeto hace su actividad con la herramienta, así como aquellos en los que el sujeto simplemente está cogiendo o dejando la herramienta. Dado que nos interesa conocer especialmente los momentos en los que se sitúa el brazo por encima de la altura del hombro, con ayuda del movimiento simulado, seleccionamos y nos centramos en aquellos instantes de tiempo que presentan esa información de interés. Estos tramos considerados suelen tener una duración aproximada de 10 segundos.

El escalado se realiza para el modelo completo, al igual que la cinemática, incluyendo ambos miembros superiores. Sin embargo, dado que todos los trabajadores analizados son diestros, la dinámica se lleva a cado teniendo en cuenta solamente los músculos de la zona derecha. Por su parte, la optimización estática, se realiza bloqueando todos los grados de libertad del modelo y dejando únicamente los tres movimientos del hombro nombrados anteriormente.

Las estimaciones obtenidas para el brazo derecho de los distintos sujetos analizados con el modelo sin el exoesqueleto y con exoesqueleto simulado se pueden apreciar en la Figura 2. 
Estimación de esfuerzos generados en el hombro por parte de trabajadores de la industria del automóvil mediante modelos biomecánicos de medidas in situ
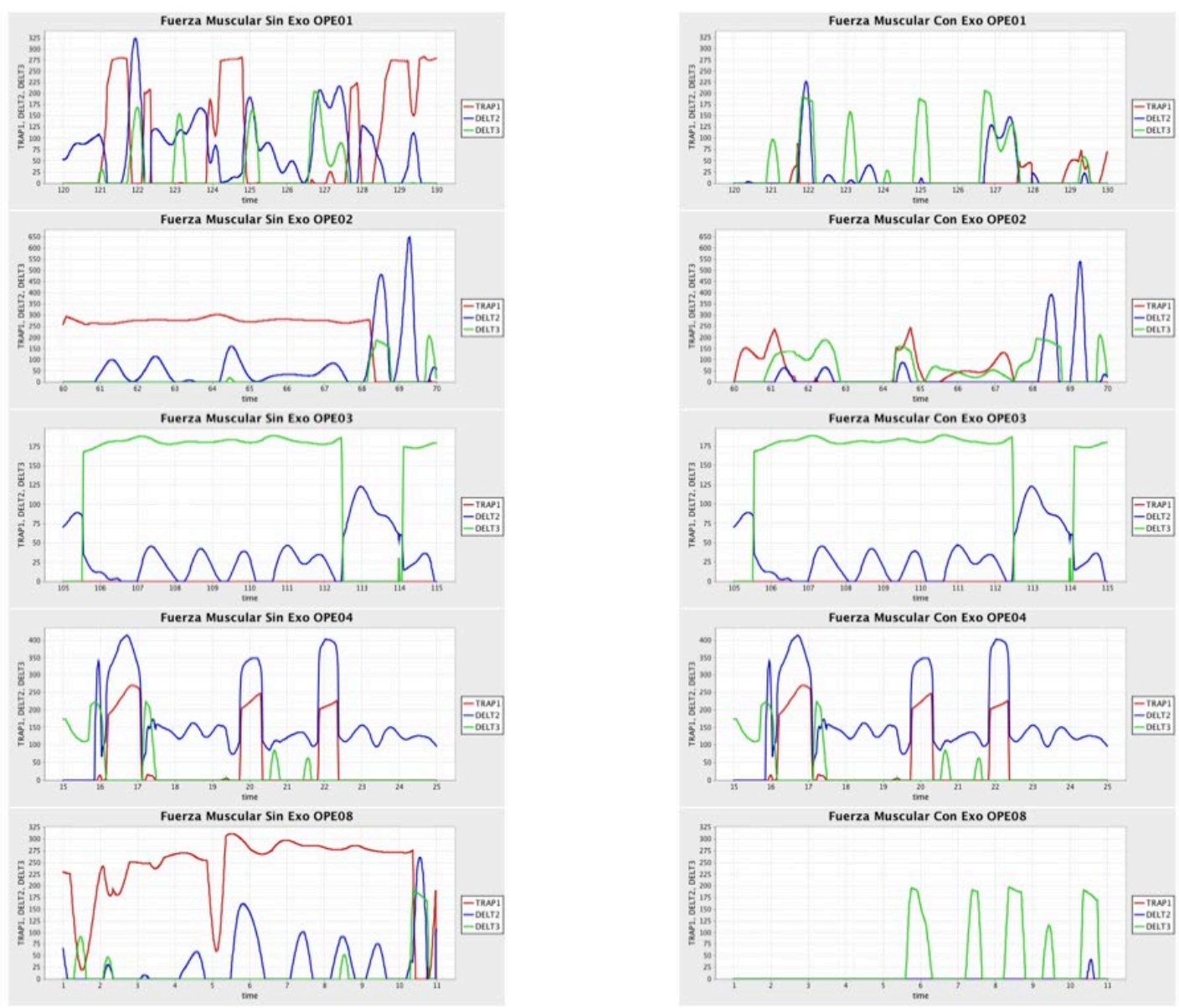

Fig. 2. Estimaciones musculares para los diferentes sujetos analizados: a la izqueirda se encuentran los sujetos con el modelo sin exoesqueleto y a la derecha con el modelo con exoesqueleto (resorte).

Fuente: Creación propia (2019).

Se aprecia que en algunos sujetos la presencia del exoesqueleto ayuda a una disminución de los esfuerzos realizados, especialmente dicha reducción es signifitiva en la línea de acción TRAP1 del músculo trapecio, el cual en ocasiones parece descender su esfuerzo en un 90 $95 \%$, tomando valores cercanos al cero, como en el caso del sujeto OPE01. En este caso, los esfuerzos de los músculos TRAP1 y DELT2 se ven atenuados significativamente, mientras que el DELT3 parece tomar los mismos valores e incluso valores ligeramente más elevados. En la mayoría de casos parece observarse que cuando los sujetos no presentan exoesqueleto los valores de esfuerzo en el DELT2 son mucho mayores que para el DELT3, 
pero una vez se tienen en cuenta el exoesqueleto la situación se invierte y es el DELT3 el que pasa a tener valores del mismo orden o incluso mucho mayores.

De la misma manera, sucede con el sujeto OPE02 y OPE08, en el que nuevamente los TRAP1 y DELT2 disminuyen, pero el DELT3 aumenta, aunque en estos casos a valores mucho más elevados que los que presentaban inicialmente. En OPE08 el TRAP1 parece incluso dejar de realizar un esfuerzo y tomar un valor constante de cero. Por otra parte, algunos sujetos como OPE03 y OPE04, la presencia del exoesqueleto no supone ninguna mejora en la reducción de los esfuerzos, siendo incluso idénticos tanto con él como sin él.

Finalmente, los percentiles p20, p50 y p75 obtenidos para los valores de los esfuerzos musculares de las líneas musculares TRAP1, DELT2 y DELT3 se recogen en la Tabla 3.

Tabla 3. Percentiles p20, p50 y p75 para diferentes líneas de acción musculares. Se resaltan en color rojo los aumentos en los valores de esfuerzos musculares.

\begin{tabular}{|c|c|c|c|c|c|c|c|c|c|c|}
\hline \multirow[b]{2}{*}{ Percentiles } & & \multicolumn{3}{|c|}{ TRAP1 } & \multicolumn{3}{|c|}{ DELT2 } & \multicolumn{3}{|c|}{ DELT3 } \\
\hline & & P25 & P50 & P75 & P25 & P50 & P75 & P25 & P50 & P75 \\
\hline \multirow[t]{2}{*}{ OPE01 } & Sin Exo & 0,001 & 0,040 & 214,549 & 14,207 & 81,117 & 116,967 & 0,000 & 0,000 & 2,337 \\
\hline & Con Exo & 0,000 & 0,000 & 0,007 & 0,000 & 0,000 & 5,841 & 0,000 & 0,000 & 53,683 \\
\hline \multirow[t]{2}{*}{ OPE02 } & Sin Exo & 264,148 & 275,974 & 278,974 & 0,127 & 29,417 & 76,314 & 0,000 & 0,000 & 0,000 \\
\hline & Con Exo & 0,000 & 0,004 & 65,881 & 0,000 & 0,000 & 5,321 & 0,000 & 44,599 & 120,148 \\
\hline \multirow[t]{2}{*}{ OPE03 } & Sin Exo & 0,000 & 0,000 & 0,000 & 7,550 & 26,502 & 43,643 & 172,768 & 180,055 & 182,222 \\
\hline & Con Exo & 0,000 & 0,000 & 0,000 & 7,550 & 26,502 & 43,643 & 172,768 & 180,055 & 182,222 \\
\hline \multirow[t]{2}{*}{ OPE04 } & Sin Exo & 0,000 & 0,000 & 4,014 & 111,188 & 130,947 & 158,363 & 0,000 & 0,000 & 0,000 \\
\hline & Con Exo & 0,000 & 0,000 & 4,014 & 111,188 & 130,947 & 158,363 & 0,000 & 0,000 & 0,000 \\
\hline \multirow[t]{2}{*}{ OPE05 } & Sin Exo & 0,001 & 226,936 & 273,209 & 0,000 & 0,001 & 23,133 & 0,000 & 0,000 & 0,000 \\
\hline & Con Exo & 0,000 & 0,000 & 0,000 & 0,000 & 0,000 & 0,000 & 0,000 & 0,000 & 10,579 \\
\hline
\end{tabular}

Con los percentiles se puede observar de forma cuantitativa, los mismos resultados obtenidos a partir de las gráficas. Para los operarios OPE01, OPE02 y OPE08, se identifican ciertas disminuciones cuando a los sujetos se les considera la colocación de un exoesqueleto, en las líneas musculares TRAP1 y DELT2; mientras que para el DELT3 se observa un aumento del esfuerzo realizado pasando de valores cercanos a cero a valores 
Estimación de esfuerzos generados en el hombro por parte de trabajadores de la industria del automóvil mediante modelos biomecánicos de medidas in situ

relativamente elevados: 53,683, 120,148 y 10,579, respectivamente. De igual manera, se comprueba que para los sujetos, OPE03 y OPE04, la colocación del exoesqueleto no supone ningún cambio en los valores de las estimaciones musculares.

\section{Discusión y conclusiones}

La análisis realizado con OPENSIM para los diferentes trabajadores considerados presenta discrepancias en los resultados obtenidos y puede que incluso contradictorios, pues en parte de ellos resulta que al comparar un movimiento, con el correspondiente esfuerzo que conlleva, con y sin un exoesqueleto que ayude a la realización de la tarea, efectivamente disminuyen los valores esfuerzo estimado en algunas de las líneas de acción que conforman los músculos principales del movimiento del hombro. Pero no afecta a todas ellas de la misma manera, ya que con el DELT3 pasa justo lo contrario y se ve aumentado. Mientras, en los otros, la presencia del exoesqueleto no supone cambios en los valores, no afecta el hecho de que un elemento de ayuda presente modifique el esfuerzo realizado por el trabajador.

Sin embargo, habría que tener algunas consideraciones en estos resultados para intentar explicar de alguna manera la diversidad que representan. Como se ha explicado en la metodología, los instantes de tiempo considerados son aquellos en los que los sujetos presentaban los brazos por encima de la línea del hombro y con la herramienta en la mano derecha. Dada esta posición es lógico, entonces, que el DELT2, por su localización anatómica anterior presente valores de activación mayores y mantenidos que en el DELT3, pues en este caso al tener los brazos ( $\sin$ ayuda de exoesqueleto) arriba supone un movimiento combinado de flexión-extensión y aducción-abducción, dado en parte por la contracción de la porción del deltoides anterior (DELT2), y por el TRAP1, que de igual forma colabora con la elevación del brazo incluso con una participación mayor. Por esta misma razón, cuando introducimos un elemento que simula el exoesqueleto y afectamos al movimiento "shoulder_elv", la contracción del mismo, así como la porción del trapecio, se ven disminuídas considerablemente, pues el resorte está ayudando al músculo a ejecer un esfuerzo de elevación del brazo y mantenerlo arriba. El efecto contrario sucede con el DELT3, el cual tira del brazo hacia abajo continuamente cuando se contrae y el hecho de que haya un elemento que lo eleva, provoca una mayor activación por su parte.

Ante esta situación, una posible manera de conseguir una disminución de todos los grupos musculares podría ser considerando otro tipo de elemento, y no simplemente un resorte, que a su vez ayude a la elevación de los brazos, y también permita una bajada amortiguada y suave, evitando que algunos músculos tengan que realizar un esfuerzo superior que en el que se realiza en una situación normal. 
En cuanto a los sujetos en los que el exoesqueleto no afecta al esfuerzo realizado, este hecho es debido a que, posiblemente, en los instantes considerados los movimientos realizados por los operarios no se llegue a entrar en el rango o a superar el límite con los que el resorte ha sido definido para actuar. De esta manera, al no verse dentro del rango de actuación determinado, el resorte no actúa y, por tanto, es como si el sujeto no llevase ningún elemento de ayuda y los valores de esfuerzos se mantienen iguales en ambos casos. Esto quiere decir, que aún con la presencia del exoesqueleto existen posturas en las que no parece aportar ningún tipo de ayuda a la realización de un trabajo concreto, generando situaciones en las que apoya y en las que no. Situaciones en las que también puede existir una postura o esfuerzo de riesgo para el paciente, y que el exoesqueleto no puede impedir, pues su actuación está limitada.

Finalmente, OPENSIM como una primera aproximación a una herramienta de evaluación de riesgos parece ser un buen método para llevar a cabo estimaciones de esfuerzos musculares, pues los resultados que se pueden extraer de él se acoplan de mejor o peor manera a la realidad, aunque se pueden explicar correctamente. No obstante, a la hora de realizar todo un análisis de estimaciones hay que tener en cuenta las limitaciones que puede presentar el programa y lo poco robusto que puede llegar a ser. Aún así, los resultados obtenidos, a pesar de las limitaciones y posibles simplificaciones en los modelos, son consistentes con los esfuerzos realizados realmente y pueden ayudar a determinar aquellas situaciones de riesgo para los trabajadores.

En estudios que requieran resultados más exactos y más realistas, sería conveniente no considerar simplificaciones, disponiendo de un modelo mucho más completo, con toda la anatomía involucrada en el movimiento total; realizar el estudio para muchos más instantes de tiempo, incluso que se estudie un ciclo de trabajo entero; y, disponer, de un elemento simulador de exoesqueleto con más variedad de características y restricciones que se pueden ajustar a una reproducción más fidedigna de un exoesqueleto real.

\section{Referencias}

DELP, S y col. (2007). "OpenSim: Open-Source Software to Create and Analyze Dynamic Simulations of Movement" en Biomedical Engineering, IEEE Transactions, vol. 54, p. 1940-50. DOI: 10.1109/TBME.2007.901024.

DE VICENTE, Á y col. (2012). "El transtorno Musculoesquelético en el ámbito laboral en cifras" en Departamento de Información e Investigación del Instituto Nacional de Seguridad e Higiene en el Trabajo, NIPO: 272-13-027-7.

FÉLIX, M. (2011). “Tareas repititivas II: Evaluación del riesgo para 1 aextremidad superior” en Instituo Nacional de Seguridad e Higiene en el Trabajo, Centro Nacional de Nuevas Tecnologías. 
Estimación de esfuerzos generados en el hombro por parte de trabajadores de la industria del automóvil mediante modelos biomecánicos de medidas in situ

HIGNETT, S y MCATAMNEY, L. (2000). "Rapid entire body assessnment (REBA)" en Applied Ergonomics, vol. 31, p. 201-5. DOI: 10.1016/S0003-6870(99)00039-3.

INSTITUTO DE BIOMECÁNICA. Ergo/IBV - Software Evaluación de Riesgos Ergonómicos. $<$ https://www.ibv.org/productos-y-servicios/productos/aplicaciones-tic/ergoibv-software-evaluacionde-riesgos-ergonomicos $>$ [Consulta: 23-Abril-2019]

JONSSON, B. (1982). "Measurement and evaluation of local muscular strain in the shoulder during constrained work" en Journal of human ergology, vol. 11, p. 73-88. DOI: 10.11183/jhe1972.11.73.

KAPANDJI, I. A. (1982). Cuadernos de fisiologí-a articular : esquemas comentados de mecánica articular. Cuaderno I, Miembro superior. El hombro. El codo. Pronosupinación. La muñeca. La mano y los dedos. Barcelona: Toray-Masson, Edi. 4. ISBN: 8431101598.

NCSRR (The National Center for Simulation in Rehanilitation Research). OpenSim Documentation. $<$ https://simtk-confluence.stanford.edu:8443/display/OpenSim/Documentation> [Consulta: 30-Abril2019]

RAMÍREZ MUÑOZ, R (1993). La industria de la automoción: su evolución e incidencia social y económica. Madrid: Editorial Complutense.

SAUL, K y col. (2014). "Benchmarking of dynamic simulation predictions in two software platforms using an upper limb musculoskeletal model" en Computer methods in biomechanics and biomedical engineering, vol. 18, p. 1-14. DOI: 10.1080/10255842.2014.916698.

WU, G y col. (2005). "ISB recommendation on definitions of joint coordinate systems of various joints for the reporting of human joint motion - Part II: Shoulder, elbow, wrist and hand" en Journal of biomechanics, vol. 38, p. 981-92. DOI: 10.1016/j.jbiomech.2004.05.042.

XSENS. Xsens MVN Analyze. <https://www.xsens.com/products/xsens-mvn-analyze/> [Consulta: 23Abril-2019]

ZHOU, H. y col. (2006). "Inertial measurements of upper limb motion" en Medical \& Biological Engineering \& Computing, vol. 44, p. 479-87. DOI: 10.1007/s11517-006-0063-z. 